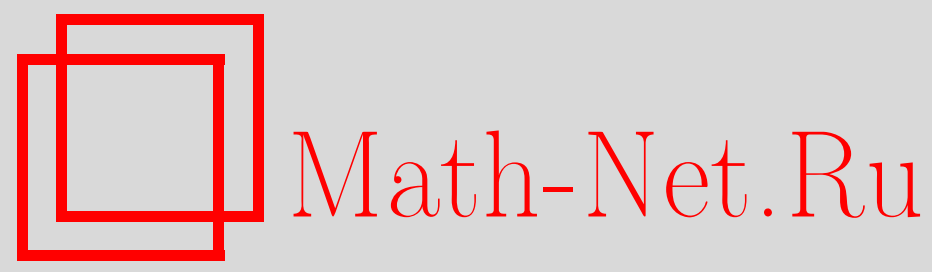

С. В. Смышляев, Совершенно уравновешенные дискретные функции и условие Голича, Дискрет. матем., 2013, том 25, выпуск 1, 63-75

DOI: https://doi.org/10.4213/dm1221

Использование Общероссийского математического портала Math-Net.Ru подразумевает, что вы прочитали и согласны с пользовательским соглашением http://www.mathnet.ru/rus/agreement

Параметры загрузки:

IP : 54.89 .56 .158

26 апреля 2023 г., 10:02:34 


\title{
Совершенно уравновешенные дискретные функции и условие Голича
}

\author{
() 2013 г. С. В. Смышляев
}

\begin{abstract}
Рассматриваются вопросы применимости гипотезы Голича в $k$-значном случае. Для простых $k$ получен ряд результатов, косвенно подтверждающих справедливость $k$-значного аналога гипотезы Голича. Полностью доказано, что в случае составных $k$ аналог гипотезы Голича вместе с определенным классом его ослаблений справедливым не является.

Работа поддержана Российским фондом фундаментальных исследований, проект 12-01-00680-a.
\end{abstract}

\section{1. Определения и предварительные результаты}

Для всяких $n \geqslant 1, k \geqslant 2$ через $E_{k}$ будем обозначать множество $\{0,1, \ldots, k-1\}$, через $P_{k}^{(n)}-$ множество $k$-значных функций $n$ переменных $\left\{f: E_{k}^{n} \rightarrow E_{k}\right\}$;

$$
P_{k}=\bigcup_{n=0}^{\infty} P_{k}^{(n)} .
$$

Для всякого натурального $l$ и всякой функции $f \in P_{k}^{(n)}$ будем рассматривать также отображение $f_{l}: E_{k}^{l+n-1} \rightarrow E_{k}^{l}$, такое, что

$$
f_{l}\left(x_{1}, x_{2}, \ldots, x_{l+n-1}\right)=\left(f\left(x_{1}, \ldots, x_{n}\right), \ldots, f\left(x_{l}, \ldots, x_{l+n-1}\right)\right) .
$$

Для всякой функции $f \in P_{k}^{(n)}$ через $\overleftrightarrow{f}$ обозначим функцию из $f \in P_{k}^{(n)}$ такую, что для всякого набора $\left(x_{1}, x_{2}, \ldots, x_{n}\right) \in E_{k}^{n}$ выполнено соотношение

$$
\overleftrightarrow{f}\left(x_{1}, x_{2}, \ldots, x_{n}\right)=f\left(x_{n}, x_{n-1}, \ldots, x_{1}\right)
$$

Определение 1. Функция $f \in P_{k}^{(n)}$ называется совершенно уравновешенной, что обозначается как $f \in \mathscr{P P B}_{k}^{(n)}$, если для всякого натурального $l$ и всякого $\mathbf{y} \in E_{k}^{l}$ верно равенство

$$
\left|f_{l}^{-1}(\mathbf{y})\right|=k^{n-1}
$$

Предложение 1 ([8, 6]). Для всякой функцฺии $f \in P_{k}^{(n)}$ верно, что функциия $\overleftrightarrow{f}$ совершенно уравновешена тогда и только тогда, когда $f$ совершенно уравновешена. 
Определение 2 ([8, 3]). Функция $f \in P_{k}^{(n)}$ называется функцией без потери информации, если при любом $l \geqslant n$ система

$$
\begin{aligned}
f_{l}\left(x_{1}, x_{2}, \ldots, x_{l+n-1}\right) & =f_{l}\left(z_{1}, z_{2}, \ldots, z_{l+n-1}\right), \\
\left(x_{1}, x_{2}, \ldots, x_{n-1}\right) & =\left(z_{1}, z_{2}, \ldots, z_{n-1}\right), \\
\left(x_{l+1}, x_{l+2}, \ldots, x_{l+n-1}\right) & =\left(z_{l+1}, z_{l+2}, \ldots, z_{l+n-1}\right), \\
\left(x_{n}, x_{n+1}, \ldots, x_{l}\right) & \neq\left(z_{n}, z_{n+1}, \ldots, z_{l}\right)
\end{aligned}
$$

является несовместной.

Теорема 1 ([8, 3]). Пусть $f \in P_{k}^{(n)}$. Следующие утверждения эквивалентны:

(1) $f$ является совершенно уравновешенной функиией;

(2) $f$ является функцией без потери информации.

Определение 3. Функция $f \in P_{k}^{(n)}$ имеет правый барьер длины $b, b \geqslant 1$, если из равенства

$$
f_{b}\left(x_{1}, x_{2}, \ldots, x_{n-1}, x_{n}^{\prime}, \ldots, x_{b+n-1}^{\prime}\right)=f_{b}\left(x_{1}, x_{2}, \ldots, x_{n-1}, x_{n}^{\prime \prime}, \ldots, x_{b+n-1}^{\prime \prime}\right)
$$

следует равенство

$$
x_{n}^{\prime}=x_{n}^{\prime \prime}
$$

Функция $f \in P_{k}^{(n)}$ имеет левый барьер длины $b, b \geqslant 1$, что обозначается как $b_{f}^{L}=b$, если функция $\overleftrightarrow{f}\left(x_{1}, x_{2}, \ldots, x_{n}\right)$ имеет правый барьер длины $b$.

Функция $f \in P_{k}^{(n)}$ имеет барьер, если она имеет правый или левый барьер, или оба сразу. При этом длиной барьера функции называется, соответственно, длина правого барьера, левого барьера, или меньшая из длин барьеров.

Абсолютно аналогично случаю $k=2$ (см. [4]), для произвольного $k \geqslant 2$ в $P_{k}$ доказываются следующие утверждения.

Предложение 2. Если функизия $f \in P_{k}^{(n)}$ имеет барьер, то она является функцией без потери информаџии.

Следствие 1. Если функция $f \in P_{k}^{(n)}$ имеет барьер, то она является совершенно уравновешенной.

Предложение 3. Функциия $f \in P_{k}^{(n)}$ имеет правыцй барьер длины 1 тогда и только тогда, когда является перестановочной по последней переменной.

Предложение 4. Если функция $f \in P_{k}^{(n)}$ имеет правый барьер длины $b$, то для произвольного $n^{\prime} \geqslant \max \{0, b-n+1\}$ и любой функциии $f^{\prime} \in P_{k}^{\left(n^{\prime}+n-b\right)}$ верно, что функция

$$
g\left(x_{1}, \ldots, x_{n^{\prime}+n}\right) \equiv f^{\prime}\left(x_{1}, \ldots, x_{n^{\prime}+n-b}\right)+f\left(x_{n^{\prime}+1}, \ldots, x_{n^{\prime}+n}\right)
$$

имеет правый барьер длины $b$.

Для всякого $s \geqslant 1$ и всякой функции $f \in P_{k}^{(n)}$ будем обозначать через $f^{(s)}$ функцию из $P_{k}^{(s(n-1)+1)}$

$$
f^{(s)}\left(x_{1}, x_{2}, \ldots, x_{s(n-1)+1}\right)=f\left(x_{1}, x_{1+s}, x_{1+2 s}, \ldots, x_{1+(n-1) s}\right) .
$$


Предложение 5. Для всякого $s \geqslant 1$ верно, что функиия $f \in P_{k}^{(n)}$ является совершенно уравновешенной тогда и только тогда, когда функция $f^{(s)} \in P_{k}^{(s(n-1)+1)}$ является совершенно уравновешенной.

Предложение 6. Для всякого $s \geqslant 1$ верно, что функиия $f \in P_{k}^{(n)}$ имеет правый барьер длины $b$ тогда и только тогда, когда функция $f^{(s)} \in P_{k}^{(s(n-1)+1)}$ имеет правый барьер длиньл $s(b-1)+1$.

\section{2. Основные результаты}

Голичем в работе [2] в 1996 году рассматривался (при $k=2$ ) вопрос о важном для используемых в фильтрующих генераторах функций свойстве, являющемся естественным усилением свойства совершенной уравновешенности.

Определение 4. Функция $f \in \mathscr{P g}_{k}^{(n)}$ называется сильно совершенно уравновешенной, если при добавлении любого числа фиктивных переменных между существенными она сохраняет совершенную уравновешенность.

Нетрудно показать, что перестановочность дискретной функции по первой или последней существенной переменной влечет за собой сильную совершенную уравновешенность. Голичем в работе [2] было предположено, что верно и обратное - данная гипотеза позже, начиная с работы Дихтла [1], стала называться гипотезой Голича.

Гипотеза 1 ([2]). При $k=2$ из сильной совершенной уравновешенности некоторой $k$-значной функции следует ее перестановочность (т.е. линейность) по первой или последней существенной переменной.

Косвенное подтверждение справедливости гипотезы Голича было впервые получено в 2008 г. в [4]. В [5] в 2012 г. гипотеза Голича была полностью доказана. Таким образом, известно, что невозможно построить кодирующее устройство на основе регистра сдвига и фильтрующей функции (над алфавитом $\{0,1\}$ ), обеспечивающее сохранение истинной случайности битовой последовательности при любом выборе точек входа (см. [2]). Интересен вопрос о возможности построения таких кодирующих устройств над алфавитом большей мощности - в особенности, в случае алфавита мощности $k=2^{m}, m \geqslant 2$, т.е. в случае рассмотрения кодирующих устройств, преобразующих входную битовую последовательность блоками длины $m$.

Сформулируем условие Голича для случая произвольного $k \geqslant 2$.

Условие Голича. Сильная совершенная уравновешенность в $P_{k}$ эквивалентна перестановочности по первой или последней существенной переменной.

Из результатов работ $[2,5]$ следует, что условие Голича выполнено в $P_{2}$. Настоящая работа посвящена рассмотрению следующего обобщения гипотезы Голича, связывающего справедливость условия Голича в $P_{k}$ с простотой числа $k$.

Гипотеза 2. Условие Голича выполнено в $P_{k}$ тогда и только тогда, когда $k-$ простое число.

Рассмотрим вначале случай составного $k$.

Теорема 2. Для всякого составного $k$ существует функция $g \in P_{k}$ с правым барьером длинь 2, существенно зависящая от последней переменной и не перестановочная по ней. 
Доказательство. Пусть $k=p q, p>1, q>1$. Рассмотрим функцию из $P_{k}^{(2)}$

$$
g(x, y)=(x \bmod p) q+\left\lfloor\frac{y}{p}\right\rfloor \bmod k .
$$

Легко видеть, что данная функция существенно зависит от обеих переменных и не является перестановочной ни по одной из них.

Чтобы доказать, что $g$ имеет правый барьер длины 2, достаточно проверить несовместность системы

$$
\begin{aligned}
g\left(x, y_{1}\right) & =g\left(x, z_{1}\right), \\
g\left(y_{1}, y_{2}\right) & =g\left(z_{1}, z_{2}\right), \\
y_{1} & \neq z_{1} .
\end{aligned}
$$

Нетрудно убедиться, что для данной системы имеет место эквивалентность

$$
\begin{aligned}
& (x \bmod p) q+\left\lfloor\frac{y_{1}}{p}\right\rfloor \bmod k=(x \bmod p) q+\left\lfloor\frac{z_{1}}{p}\right\rfloor \bmod k, \\
& \left(y_{1} \bmod p\right) q+\left\lfloor\frac{y_{2}}{p}\right\rfloor \bmod k=\left(z_{1} \bmod p\right) q+\left\lfloor\frac{z_{2}}{p}\right\rfloor \bmod k, \\
& y_{1} \neq z_{1} \\
& \mathbb{1} \\
& \left\lfloor\frac{y_{1}}{p}\right\rfloor \bmod k=\left\lfloor\frac{z_{1}}{p}\right\rfloor \bmod k, \\
& \left(y_{1}-z_{1} \bmod p\right) q \bmod k=\left\lfloor\frac{z_{2}}{p}\right\rfloor-\left\lfloor\frac{y_{2}}{p}\right\rfloor \bmod k, \\
& y_{1} \neq z_{1} \text {. }
\end{aligned}
$$

Из первого равенства данной системы следует неравенство

$$
\left|y_{1}-z_{1}\right| \leqslant p-1
$$

откуда, с учетом неравенства $y_{1} \neq z_{1}$ следует неравенство

$$
y_{1}-z_{1} \bmod p \geqslant 1 \text {. }
$$

С учетом (3), из (2) получим

$$
\begin{aligned}
& \left\lfloor\frac{z_{2}}{p}\right\rfloor-\left\lfloor\frac{y_{2}}{p}\right\rfloor \bmod k \geqslant q, \\
& \left\lfloor\frac{z_{2}}{p}\right\rfloor-\left\lfloor\frac{y_{2}}{p}\right\rfloor \bmod k \leqslant(p-1) q .
\end{aligned}
$$

С другой стороны, для всякого $z \in E_{k}$ выполнено неравенство

$$
0 \leqslant\left\lfloor\frac{z}{p}\right\rfloor \leqslant q-1,
$$

а следовательно, либо

$$
\left\lfloor\frac{z_{2}}{p}\right\rfloor-\left\lfloor\frac{y_{2}}{p}\right\rfloor \bmod k=0,
$$


либо

$$
1 \leqslant\left\lfloor\frac{z_{2}}{p}\right\rfloor-\left\lfloor\frac{y_{2}}{p}\right\rfloor \bmod k \leqslant q-1,
$$

либо

$$
k-1 \geqslant\left\lfloor\frac{z_{2}}{p}\right\rfloor-\left\lfloor\frac{y_{2}}{p}\right\rfloor \bmod k \geqslant k-(q-1)=(p-1) q+1 .
$$

Таким образом, в любом из случаев условия системы (4) не выполняются, а значит, система (2) и исходная система (1) несовместны. Следовательно, функция $g$ имеет правый барьер длины 2, и теорема полностью доказана.

Следствие 2. Для всякого составного $k$ существует функиия $f \in \mathscr{P פ B}_{k}^{(2)}$, сущчественно зависящая от обеих переменных и не являющаяся по ним перестановочной.

Для доказательства следствия достаточно воспользоваться следствием 1.

Следствие 3. Если $k$ - составное, то условие Голича не выполнено в $P_{k}$.

Доказательство. С учетом следствия 1 получим, что при добавлении произвольного числа фиктивных переменных совершенно уравновешенной функции двух переменных всегда строится совершенно уравновешенная функция, иными словами, любая совершенно уравновешенная функция двух переменных является сильно совершенно уравновешенной. Учитывая следствие 2 , получим, что для любого составного $k$ в $P_{k}$ существует сильно совершенно уравновешенная функция двух переменных, существенно зависящая от обеих переменных и не являющаяся по ним перестановочной. Таким образом, при всяком составном $k$ условие Голича не выполняется.

Теорема 3. Для всякого составного $k$ и всякого $n \geqslant 2$ существует сильно совершенно уравновешенная функиия $f \in P_{k}^{(n)}$, существенно зависящая от всех $n$ переменных, которая не является перестановочной ни по первой, ни по последней переменной, но является сильно совершенно уравновешенной.

Доказательство. Для доказательства утверждения в случае $n=2$ достаточно воспользоваться теоремой 2.

Пусть $n \geqslant 3$. Рассмотрим функцию $g \in P_{k}^{(2)}$ с правым барьером длины 2, построенную в ходе доказательства теоремы 2. Данная функция существенно зависит от обеих переменных и не является перестановочной ни по одной из них. Рассмотрим функцию $f \in P_{k}^{(n)}$

$$
f\left(x_{1}, x_{2}, \ldots, x_{n}\right)=x_{1}^{2}+x_{2}^{2}+\ldots+x_{n-2}^{2}+g\left(x_{n-1}, x_{n}\right) .
$$

Данная функция, в соответствии с предложением 4, имеет правый барьер длины 2. Очевидно, что она существенно зависит от всех $n$ переменных и не является перестановочной ни по одной из них. Покажем, что данная функция является сильно совершенно уравновешенной.

Рассмотрим произвольную функцию $\tilde{f} \in P_{k}^{(N)}$, получаемую из $f$ добавлением фиктивных переменных,

$$
\tilde{f}\left(x_{1}, x_{2}, \ldots, x_{N}\right)=f\left(x_{\varphi(1)}, x_{\varphi(2)}, \ldots, x_{\varphi(n)}\right),
$$


где $\varphi:\{1,2, \ldots, n\} \rightarrow\{1,2, \ldots, N\}, \varphi(i+1)>\varphi(i)$ для всякого $i=1,2, \ldots, n-1$. Тогда функция

$$
\tilde{f}\left(x_{1}, x_{2}, \ldots, x_{N}\right)+\left(-x_{\varphi(1)}^{2}-x_{\varphi(2)}^{2}-\ldots-x_{\varphi(n-2)}^{2}\right)=g\left(x_{\varphi(n-1)}, x_{\varphi(n)}\right),
$$

как следует из предложения 6 , имеет правый барьер длины

$$
(N-\varphi(n))+(1+\varphi(n)-\varphi(n-1))=N+1-\varphi(n-1),
$$

тогда, по предложению 4 , и функция $\tilde{f}$ имеет правый барьер длины $N+1-\varphi(n-1)$. Таким образом, $\tilde{f}$ является совершенно уравновешенной.

Итак, всякая функция, получаемая из $f$ добавлением фиктивных переменных, является совершенно уравновешенной, а значит, $f$ является сильно совершенно уравновешенной, что и завершает доказательство утверждения.

Рассмотрим теперь случай простого $k$. Воспользуемся следующим утверждением, верным для произвольного $k \geqslant 2$ и доказываемым абсолютно аналогично теореме 3 в [7].

Лемма 1. Пусть $k \geqslant 2$. Для любой функции $f \in P_{k}^{(n)}$ c правым барьером длины $b$ существует такое число $c, c=c(f)$, что для любого $l \geqslant b-1$ и любых наборов $\left(x_{1}, x_{2}, \ldots, x_{n-1}\right) \in E_{k}^{n-1},\left(y_{1}, y_{2}, \ldots, y_{l}\right) \in E_{k}^{l}$ выполняется одно из двух равенств:

$$
\left|f_{l}\left(x_{1}, x_{2}, \ldots, x_{n-1}, \cdot, \cdot, \ldots, \cdot\right)^{-1}\left(y_{1}, y_{2}, \ldots, y_{l}\right)\right|=0
$$

или

$$
\left|f_{l}\left(x_{1}, x_{2}, \ldots, x_{n-1}, \cdot, \cdot, \ldots, \cdot\right)^{-1}\left(y_{1}, y_{2}, \ldots, y_{l}\right)\right|=c
$$

Теорема 4. Пусть $k$ - простое число, и функция $f \in P_{k}^{(n)}$ имеет правый барьер длинь 2. Тогда функция $f$ не зависит существенно от последней переменной и перестановочна по предпоследней.

Доказательство. В соответствии с леммой 1 , для некоторого натурального $c$ верно, что для любого набора $\left(x_{1}, x_{2}, \ldots, x_{n-1}\right) \in E_{k}^{n-1}$ и всякого $y_{1} \in E_{k}$ выполнено либо

$$
\left|f\left(x_{1}, x_{2}, \ldots, x_{n-1}, \cdot\right)^{-1}\left(y_{1}\right)\right|=c,
$$

либо

$$
\left|f\left(x_{1}, x_{2}, \ldots, x_{n-1}, \cdot\right)^{-1}\left(y_{1}\right)\right|=0
$$

С другой стороны, очевидно равенство

$$
\sum_{y_{1} \in E_{k}}\left|f\left(x_{1}, x_{2}, \ldots, x_{n-1}, \cdot\right)^{-1}\left(y_{1}\right)\right|=\left|E_{k}\right|=k,
$$

верное при всяком $\left(x_{1}, x_{2}, \ldots, x_{n-1}\right) \in E_{k}^{n-1}$. Получаем, что

$$
c \mid \operatorname{Im}\left(f\left(x_{1}, x_{2}, \ldots, x_{n-1}, \cdot\right) \mid=k,\right.
$$

откуда ввиду простоты $k$ следует, что либо $c=1$, либо $c=k$. При $c=1$ получаем, что

$$
\mid \operatorname{Im}\left(f\left(x_{1}, x_{2}, \ldots, x_{n-1}, \cdot\right) \mid=k\right.
$$


для всякого $\left(x_{1}, x_{2}, \ldots, x_{n-1}\right) \in E_{k}^{n-1}$, что соответствует перестановочности по последней переменной, то есть наличию правого барьера длины 1, что противоречит условию. При $c=k$ получаем, что

$$
\mid \operatorname{Im}\left(f\left(x_{1}, x_{2}, \ldots, x_{n-1}, \cdot\right) \mid=1\right.
$$

для всякого $\left(x_{1}, x_{2}, \ldots, x_{n-1}\right) \in E_{k}^{n-1}$, что означает отсутствие существенной зависимости от последней переменной, откуда, с учетом наличия правого барьера длины 2, следует перестановочность по предпоследней переменной.

Следствие 4. При простом $k$ условие Голича не нарушается на функциях с барьером длины 2.

Доказательство. В соответствии с теоремой 4, при простом $k$ не существует функций с барьером длины 2, существенно зависящих от обеих крайних переменных.

Из теоремы 2 и следствия 4 вытекает следующий критерий.

Следствие 5. Условие Голича не нарушается на функциях с барьером длины 2 в $P_{k}$ тогда и только тогда, когда $k$ - простое число.

Теорема 5. Пусть $k$ - простое число и функиия $f \in P_{k}^{(2)}$ имеет барьер. Тогда она перестановочна по одной из переменных.

Доказательство. Будем считать, для определенности, что функция $f \in P_{k}^{(2)}$ имеет правый барьер длины $b$. В соответствии с леммой 1 , для некоторого натурального $c$ верно, что для любого $x_{1} \in E_{k}$ и всякого набора $\left(y_{1}, y_{2}, \ldots, y_{b-1}\right) \in E_{k}^{b-1}$ выполнено либо

$$
\left|f_{b-1}\left(x_{1}, \cdot, \ldots, \cdot\right)^{-1}\left(y_{1}, y_{2}, \ldots, y_{b-1}\right)\right|=c,
$$

либо

$$
\left|f_{b-1}\left(x_{1}, \cdot, \ldots, \cdot\right)^{-1}\left(y_{1}, y_{2}, \ldots, y_{b-1}\right)\right|=0 \text {. }
$$

С другой стороны, очевидно равенство

$$
\sum_{\left(y_{1}, y_{2}, \ldots, y_{b-1}\right) \in E_{k}^{b-1}}\left|f_{b-1}\left(x_{1}, \cdot, \ldots, \cdot\right)^{-1}\left(y_{1}, y_{2}, \ldots, y_{b-1}\right)\right|=\left|E_{k}^{b-1}\right|=k^{b-1},
$$

верное при всяком $x_{1} \in E_{k}$. Получаем, что

$$
c \mid \operatorname{Im}\left(f_{b-1}\left(x_{1}, \cdot, \ldots, \cdot\right) \mid=k^{b-1},\right.
$$

откуда, ввиду простоты $k$, следует, что $c$ является некоторой степенью $k$. Так как $f$ имеет барьер, то, по следствию 1, она является совершенно уравновешенной, а значит,

$$
\left|f_{b-1}^{-1}\left(y_{1}, y_{2}, \ldots, y_{b-1}\right)\right|=k
$$

для всякого $\left(y_{1}, y_{2}, \ldots, y_{b-1}\right) \in E_{k}^{b-1}$, откуда $c \leqslant k$, то есть $c=1$ или $c=k$.

Пусть $c=1$. Если функция $f$ не является перестановочной по второй переменной, то, очевидно, отображение $f_{b-1}$ не всегда меняет значение при фиксации первых $b-1$ 
переменных и изменении последней. Другими словами, существует $x_{1} \in E_{k}$ и такой набор $\left(y_{1}, y_{2}, \ldots, y_{b-1}\right) \in E_{k}^{b-1}$, что

$$
\left|f_{b-1}\left(x_{1}, \cdot \ldots, \cdot\right)^{-1}\left(y_{1}, y_{2}, \ldots, y_{b-1}\right)\right|>1=c,
$$

чего быть не может.

Пусть теперь $c=k$. Если $f$ не является перестановочной по первой переменной, то существуют наборы $\left(\tilde{x}_{2}, \tilde{x}_{3}, \ldots, \tilde{x}_{b-1}\right) \in E_{k}^{b-1}, x_{1}^{\prime}, x_{1}^{\prime \prime} \in E_{k}, x_{1}^{\prime} \neq x_{1}^{\prime}$, такие, что

$$
f_{b-1}\left(x_{1}^{\prime}, \tilde{x}_{2}, \tilde{x}_{3}, \ldots, \tilde{x}_{b-1}\right)=f_{b-1}\left(x_{1}^{\prime \prime}, \tilde{x}_{2}, \tilde{x}_{3}, \ldots, \tilde{x}_{b-1}\right)=\left(\tilde{y}_{1}, \tilde{y}_{2}, \ldots, \tilde{y}_{b-1}\right) .
$$

Тогда

$$
\begin{aligned}
\left|f_{b-1}^{-1}\left(\tilde{y}_{1}, \tilde{y}_{2}, \ldots, \tilde{y}_{b-1}\right)\right| \geqslant & \left|f_{b-1}\left(x_{1}^{\prime}, \cdot, \ldots, \cdot\right)^{-1}\left(y_{1}, y_{2}, \ldots, y_{b-1}\right)\right| \\
& +\left|\left\{\left(x_{1}^{\prime}, \tilde{x}_{2}, \tilde{x}_{3}, \ldots, \tilde{x}_{b-1}\right)\right\}\right| \\
=c & +1=k+1,
\end{aligned}
$$

что противоречит совершенной уравновешенности $f$. Таким образом, $f$ перестановочна по первой переменной.

Следствие 6. Условие Голича не нарушается на функияях с барьером в $P_{k}^{(2)}$ тогда $u$ только тогда, когда $k$ - простое число.

Рассмотрим далее функции из $P_{k}^{(2)}$ при некоторых фиксированных $k$. В дальнейших утверждениях разрабатывается аппарат исследования функций двух переменных в $P_{k}$, который позволяет доказать справедливость условия Голича при $k \in\{3,5,7\}$, а также представляется полезным для будущих исследований, связанных с рассмотрением аналогичного утверждения в случае произвольного простого $k$.

Теорема 6. При $k \in\{3,5,7\}$ все совершенно уравновешенные функции из $P_{k}^{(2)}$ перестановочны по первой или последней переменной.

Доказательство. Каждой функции $f \in P_{k}^{(2)}$ сопоставим матрицу $\left(a_{i, j}^{f}\right) \in E_{k}^{k \times k}$, $a_{i, j}^{f}=f(i, j), i, j=0,1, \ldots, k-1$. Условие отсутствия перестановочности $f$ по обеим переменным можно записать следующим образом:

$$
\exists i, j^{\prime}, j^{\prime \prime}, i^{\prime}, i^{\prime \prime}, j \in E_{k}, \quad i^{\prime} \neq i^{\prime \prime}, \quad j^{\prime} \neq j^{\prime \prime}: \quad a_{i, j^{\prime}}^{f}=a_{i, j^{\prime \prime}}^{f}, \quad a_{i^{\prime}, j}^{f}=a_{i^{\prime \prime}, j}^{f} .
$$

По теореме 1, отсутствие совершенной уравновешенности в таком случае эквивалентно выполнению условия: найдутся $l \in \mathbf{N}, i^{*}, i_{1}^{\prime}, i_{1}^{\prime \prime}, i_{2}^{\prime}, i_{2}^{\prime \prime}, \ldots, i_{l}^{\prime}, i_{l}^{\prime \prime}, i^{* *} \in E_{k}$, такие, что

$$
\begin{gathered}
i_{1}^{\prime} \neq i_{1}^{\prime \prime}, \\
a_{i^{*}, i_{1}^{\prime}}^{f}=a_{i^{*}, i_{1}^{\prime \prime}}^{f}, \\
a_{i_{1}^{\prime}, i_{2}^{\prime}}^{f}=a_{i_{1}^{\prime \prime}, i_{2}^{\prime \prime}}^{f}, \\
\cdots \\
a_{i_{l-1}^{\prime}, i_{l}^{\prime}}^{f}=a_{i_{l-1}^{\prime \prime}, i_{l}^{\prime \prime}}^{f}, \\
a_{i_{l}^{\prime}, i^{* * *}}^{f}=a_{i_{l}^{\prime \prime}, i^{* *}}^{f} .
\end{gathered}
$$


Для произвольно выбранной функции $f \in P_{k}^{(2)}$, введем операторы $S_{f}^{\prime}(\cdot)$ и $S_{f}(\cdot)$ из $2^{\left\{\left\{i^{*}, j^{*}\right\} \mid i^{*}, j^{*} \in E_{k}\right\}}$ в $2^{\left\{\left\{i^{*}, j^{*}\right\} \mid i^{*}, j^{*} \in E_{k}\right\}}$ следующим образом: для всякого подмножества $M \subseteq\left\{\left\{i^{*}, j^{*}\right\} \mid i^{*}, j^{*} \in E_{k}\right\}$ положим

$$
\begin{aligned}
& S_{f}^{\prime}(M)=\left\{\left\{i^{\prime}, i^{\prime \prime}\right\} \mid \exists\left\{i^{*}, i^{* *}\right\} \in M: a_{i^{*}, i^{\prime}}^{f}=a_{i^{* *}, i^{\prime \prime}}^{f}\right\}, \\
& S_{f}(M)=M \cup S_{f}^{\prime}(M) .
\end{aligned}
$$

Тогда отсутствие у $f$ совершенной уравновешенности эквивалентно существованию такого $l$, что $S_{f}^{l}\left(I_{f}\right) \cap\left\{\{i\}, i \in E_{k}\right\} \neq \varnothing$, где

$$
I_{f}=\left\{\left\{i^{\prime}, i^{\prime \prime}\right\} \mid i^{\prime} \neq i^{\prime \prime}, \exists i: a_{i, i^{\prime}}^{f}=a_{i, i^{\prime \prime}}^{f}\right\} .
$$

С учетом предложения 1 , отсутствие совершенной уравновешенности у $f$ также эквивалентно существованию такого $l$, что $S_{\overleftrightarrow{f}}^{l}(I \overleftrightarrow{f}) \cap\left\{\{i\}, i \in E_{k}\right\} \neq \varnothing$.

Как следует из теоремы 5 , любая функция из $P_{k}^{(2)}$, не являющаяся перестановочной ни по первой, ни по второй переменной, не имеет ни правого, ни левого барьера.

Таким образом, достаточно показать, что для всякой функции из $P_{k}^{(2)}, k=3,5,7$, такой, что выполнено (5), верно, что существует $l \geqslant 1$ такое, что либо для некоторого $i \in E_{k}$ верно $\{i\} \in S_{f}^{l}\left(I_{f}\right)$, либо

$$
\left|S_{f}^{l}\left(I_{f}\right)\right| \geqslant\left\lfloor\frac{1}{2}\left(\begin{array}{l}
k \\
2
\end{array}\right)\right\rfloor+1 .
$$

Действительно, если для некоторой функции $f$ для самой $f$ или для $\overleftrightarrow{f}$ выполнено первое из условий, то отсутствие совершенной уравновешенности у $f$ следует из теоремы 1. Если же и для $f$, и для $\overleftrightarrow{f}$ выполнено второе из условий, то

$$
\left|\bigcup_{l=1}^{\infty} S_{f}^{l}\right|+\left|\bigcup_{l=1}^{\infty} S_{\overleftrightarrow{f}}^{l}\right| \geqslant\left(\begin{array}{l}
k \\
2
\end{array}\right)+1
$$

откуда следует существование $l^{\prime}$ такого, что $S_{f}^{l^{\prime}} \cap S_{\overleftrightarrow{f}}^{l^{\prime}} \neq \varnothing$, что, как следует из теоремы 1 , означает отсутствие совершенной уравновешенности у функции $f$. Оставшуюся часть доказательства выделим в три леммы, для случаев $k=3,5,7$, доказывая в каждой из них, что для каждой функции $f$, удовлетворяющей (5) (то есть, в частности, такой, что $\left.I_{f} \neq \varnothing\right)$ при наложении требования невыполнения первого из условий для достаточно больших значений $l$ выполняется второе из условий.

Далее для сокращения записей для всякого подмножества $T \subseteq E_{k}$ через $f_{T}$ будем обозначать сужение функции $f$ на множество $T \times E_{k}$.

Предложение 7. Если множество А состоит из всех подмножеств мощности 2 такого множества $B \subseteq E_{k}$, что $2|B|>k$, то не существует совершенно уравновешенной функции $f \in P_{k}^{(2)}$, для которой $A \subseteq S_{f}^{l}\left(I_{f}\right)$ при некотором натуральном $l$.

Доказательство. Предположим противное: пусть $f \in P_{k}^{(2)}$ является совершенно уравновешенной функцией, для которой $A \subseteq S_{f}^{l}\left(I_{f}\right)$ при некотором натуральном $l$. В таком случае для каждого $i \in E_{k}$ и всяких $i^{\prime}, i^{\prime \prime} \in B$ выполнено $a_{i^{\prime}, i}^{f} \neq a_{i^{\prime \prime}, i}^{f}$, то есть множество 
$\left\{a_{j, i}^{f} \mid j \in B\right\}$ имеет мощность $|B|$ для всякого $i \in E_{k}$. Тогда для всяких $i_{1}, i_{2} \in E_{k}$ верно $\left\{a_{j, i_{1}}^{f} \mid j \in B\right\} \cap\left\{a_{j, i_{2}}^{f} \mid j \in B\right\} \neq \varnothing$, откуда

$$
\begin{gathered}
S_{f}(A)=\left\{\{i, j\} \mid i, j \in E_{k}, i \neq j\right\}, \\
\left|S_{f}^{l+1}\left(I_{f}\right)\right|=\left|S_{f}(A)\right|=\left(\begin{array}{l}
k \\
2
\end{array}\right)>\left\lfloor\frac{1}{2}\left(\begin{array}{l}
k \\
2
\end{array}\right)\right\rfloor+1,
\end{gathered}
$$

что противоречит совершенной уравновешенности $f$.

Предложение 8. Пусть

$$
A=\left\{\left\{i_{0}, i_{1}\right\},\left\{i_{1}, i_{2}\right\},\left\{i_{2}, i_{3}\right\}, \ldots,\left\{i_{s-2}, i_{s-1}\right\},\left\{i_{s-1}, i_{0}\right\}\right\},
$$

где $s \geqslant 3$ нечетно, а $i_{0}, i_{1}, \ldots, i_{s-1} \in E_{k}$ попарно различны. Тогда не существует совершенно уравновешенной функиии $f \in P_{k}^{(2)}$, для которой одновременно выполняются следующие два условия:

(1) сущуествуют $i^{*} \in\left\{i_{0}, i_{1}, \ldots, i_{s-1}\right\}, i^{\prime}, i^{\prime \prime} \in E_{k}, i^{\prime} \neq i^{\prime \prime}$, такие, что

$$
a_{i *, i^{\prime}}=a_{i *, i^{\prime \prime}}=a^{*}
$$

(2) для всякого $\left\{i^{\prime}, i^{\prime \prime}\right\} \in A$ и для всякого $i \in E_{k}$ выполнено равенство

$$
\left|f_{\left\{i^{\prime}, i^{\prime \prime}\right\}}^{-1}(i)\right|=2 \text {. }
$$

Доказательство. Предположим противное: описанные два условия выполняются для некоторой функции $f \in P_{k}^{(2)}$. Пусть $i^{*}=i_{j}$, то есть

$$
\left|f_{\left\{i_{j}\right\}}^{-1}\left(a^{*}\right)\right|=2 .
$$

При этом, в соответствии со вторым условием, выполнено равенство

$$
\left|f_{\left\{i_{j}, i_{j+1} \bmod s\right.}^{-1}\left(a^{*}\right)\right|=2,
$$

откуда

$$
\left|f_{\left\{i_{j+1} \bmod s\right.}^{-1}\left(a^{*}\right)\right|=0 \text {. }
$$

Далее, снова по второму условию,

$$
\left|f_{\left\{i_{j+1} \bmod s, i_{j+2} \bmod s\right.}^{-1}\left(a^{*}\right)\right|=2,
$$

откуда

$$
\left|f_{\left\{i_{j+2} \bmod s\right.}^{-1}\left(a^{*}\right)\right|=2 .
$$

Продолжая аналогично, получим, что

$$
\left|f_{\left\{i_{j+t} \bmod s\right.}^{-1}\left(a^{*}\right)\right|=2
$$

для любого четного $t$. Но при $t=s+1$ справедливо равенство $j+t \quad \bmod s=1$, поэтому

$$
\left|f_{\left\{i_{j+s+1} \bmod s\right\}}\left(a^{*}\right)\right|=2,
$$

что противоречит полученному ранее. 
Лемма 2. $В P_{3}^{(2)}$ все функици, для которых выполнено (5), не являются совершенно уравновешенными.

Доказательство. Для произвольной функции $f \in P_{3}^{(2)}$, удовлетворяющей (5), рассмотрим произвольную неупорядоченную пару $\left\{i^{\prime}, i^{\prime \prime}\right\}, i^{\prime} \neq i^{\prime \prime}$, из множества $I_{f} \neq \varnothing$. Полагая теперь

$$
B=\left\{i^{\prime}, i^{\prime \prime}\right\}, \quad A=\left\{\left\{i^{\prime}, i^{\prime \prime}\right\}\right\},
$$

получим требуемое утверждение из предложения 7.

Лемма 3. В $P_{5}^{(2)}$ все функиии, для которых выполнено (5), не являются совершенно уравновешенными.

Доказательство. Для произвольной функции $f \in P_{5}^{(2)}$, удовлетворяющей (5), рассмотрим произвольную неупорядоченную пару $\left\{i^{\prime}, i^{\prime \prime}\right\}, i^{\prime} \neq i^{\prime \prime}$, из множества $I_{f} \neq \varnothing$. Так как

$$
\sum_{i \in E_{5}}\left|f_{\left\{i^{\prime}, i^{\prime \prime}\right\}}^{-1}(i)\right|=\left|\left\{i^{\prime}, i^{\prime \prime}\right\}\right|\left|E_{5}\right|=10
$$

возможны два случая.

Если существует $i \in E_{5}$, такое, что

$$
\left|f_{\left\{i^{\prime}, i^{\prime \prime}\right\}}^{-1}(i)\right| \geqslant 3
$$

то отсутствие совершенной уравновешенности у функции $f$ следует из предложения 7.

Если же для каждого $i \in E_{5}$ выполнено

$$
\left|f_{\left\{i^{\prime}, i^{\prime \prime}\right\}}^{-1}(i)\right|=2,
$$

то, как легко видеть, либо найдутся такие попарно различные элементы $\tilde{l}_{1}, \tilde{l}_{2}, \tilde{l}_{3}$, что $\left\{\tilde{l}_{1}, \tilde{l}_{2}\right\},\left\{\tilde{l}_{2}, \tilde{l}_{3}\right\},\left\{\tilde{l}_{1}, \tilde{l}_{3}\right\} \in S_{f}\left(\left\{i^{\prime}, i^{\prime \prime}\right\}\right.$, и тогда отсутствие совершенной уравновешенности у функции $f$ следует из предложения 7 , либо

$$
S_{f}\left(\left\{i^{\prime}, i^{\prime \prime}\right\}\right)=\left\{\left\{i_{0}, i_{1}\right\},\left\{i_{1}, i_{2}\right\},\left\{i_{2}, i_{3}\right\},\left\{i_{3}, i_{4}\right\},\left\{i_{4}, i_{0}\right\}\right\}, \quad\left\{i_{0}, i_{1}, i_{2}, i_{3}, i_{4}\right\}=E_{5},
$$

и тогда требуемое утверждение следует из предложения 8.

Лемма 4. $В P_{7}^{(2)}$ все функици, для которых выполнено (5), не являются совериенно уравновеменными.

Доказательство. Для произвольной функции $f \in P_{7}^{(2)}$, удовлетворяющей (5), рассмотрим произвольную неупорядоченную пару $\left\{i^{\prime}, i^{\prime \prime}\right\}, i^{\prime} \neq i^{\prime \prime}$, из множества $I_{f} \neq \varnothing$. Так как

$$
\sum_{i \in E_{7}}\left|f_{\left\{i^{\prime}, i^{\prime \prime}\right\}}^{-1}(i)\right|=\left|\left\{i^{\prime}, i^{\prime \prime}\right\}\right|\left|E_{7}\right|=14
$$

возможны два случая.

Если существует $i \in E_{7}$, такое, что

$$
\left|f_{\left\{i^{\prime}, i^{\prime \prime}\right\}}^{-1}(i)\right| \geqslant 3
$$


то в множестве $S_{f}\left(I_{f}\right)$ присутствуют три неупорядоченные пары $\left\{i_{1}, i_{2}\right\},\left\{i_{2}, i_{3}\right\},\left\{i_{1}, i_{3}\right\}$, где $i_{1}, i_{2}, i_{3} \in E_{7}, i_{1} \neq i_{2}, i_{2} \neq i_{3}, i_{1} \neq i_{3}$. Так как

$$
\sum_{i \in E_{7}}\left|f_{\left\{i_{1}, i_{2}, i_{3}\right\}}^{-1}(i)\right|=\left|\left\{i_{1}, i_{2}, i_{3}\right\}\right|\left|E_{7}\right|=21,
$$

то либо при некотором $\tilde{l}$ верно

$$
\left|f_{\left\{i_{1}, i_{2}, i_{3}\right\}}^{-1}(\tilde{l})\right| \geqslant 4
$$

и тогда функция $f$ не является совершенно уравновешенной по предложению 7; либо для всех $i \in E_{7}$ выполнено

$$
\left|f_{\left\{i_{1}, i_{2}, i_{3}\right\}}^{-1}(i)\right|=3
$$

в последнем случае при рассмотрении графа на вершинах из множества $E_{7}$, вершины $i$ и $j$ которого соединены ребром тогда и только тогда, когда $\{i, j\} \in S_{f}^{2}\left(\left\{i_{1}, i_{2}\right\},\left\{i_{2}, i_{3}\right\},\left\{i_{1}, i_{3}\right\}\right)$, легко заметить, что степень каждой вершины в этом графе не меньше 2, причем если хотя бы у одной вершины степень равна в точности двум, то описанный граф представляет собой совокупность двух полных графов на 3 и на 4 вершинах, что невозможно по предложению 7. В случае, когда степени всех вершин не меньше 3, сумма степеней вершин графа не меньше 21, откуда следует, что число ребер графа не меньше 11 , что означает справедливость неравенства

$$
\left|S_{f}^{2}\left(\left\{i_{1}, i_{2}\right\},\left\{i_{2}, i_{3}\right\},\left\{i_{1}, i_{3}\right\}\right)\right| \geqslant\left\lfloor\frac{1}{2}\left(\begin{array}{l}
7 \\
2
\end{array}\right)\right\rfloor+1 .
$$

Рассмотрим теперь случай

$$
\left|f_{\left\{i^{\prime}, i^{\prime \prime}\right\}}^{-1}(i)\right|=2
$$

для всякого $i \in E_{7}$. Граф $\left(E_{7}, S_{f}\left(\left\{i^{\prime}, i^{\prime \prime}\right\}\right)\right)$ представляет собой объединение циклов длины не менее 2 каждый и суммарной длины 7. Случай присутствия в графе цикла длины 3 рассмотрен выше. По предложению 8 доказывается, что данный граф не представляет собой цикл длины 7, то есть состоит из двух циклов длины 5 и 2, причем, по предложению 8, для каждого $i$ на цикле длины 5 для любых $i^{*} \neq i^{* *}$ верно, что $a_{i, i *}^{f} \neq a_{i, i * *}^{f}$. С учетом сказанного выше очевидно, что достаточно далее рассмотреть только случай, когда для произвольного ребра $\left\{i_{1}, i_{2}\right\}$ в цикле длины 5 верно, что граф $\left(E_{7}, S_{f}\left(\left\{i_{1}, i_{2}\right\}\right)\right)$ снова состоит из двух циклов длины 5 и 2. Очевидно, что цикл длины 2 не может совпадать у всех данных пяти графов, а значит, хотя бы в одном из этих графов в цикле длины 5 присутствует вершина $\tilde{\imath}$ такая, что для некоторых $i^{*} \neq i^{* *}$ верно, что $a_{\tilde{l}, i *}^{f}=a_{\tilde{l}, i * *}^{f}$, что, по предложению 8 , противоречит совершенной уравновешенности функции $f$.

Следствие 7. При $k \in\{2,3,5,7\}$ условие Голича не нарушается на функичях из $P_{k}^{(2)}$.

\section{3. Заключение}

Таким образом, установлено, что условие Голича: 
(1) нарушается на функциях из $P_{k}^{(2)}$ при любом составном $k$ (теорема 2) и не нарушается на функциях из $P_{k}^{(2)}$ при $k$, равном одному из четырех наименьших простых чисел ([5] и теорема 6);

(2) нарушается на функциях из $P_{k}$ с барьером длины 2 при любом составном $k$ (теорема 2) и не нарушается на функциях из $P_{k}$ с барьером длины 2 при любом простом $k$ (следствие 5);

(3) не нарушается на функциях из $P_{k}^{(2)}$ с барьером при любом простом $k$ (следствие 6);

(4) нарушается на существенно зависящих от всех переменных функциях из $P_{k}^{(n)}$ при любом составном $k$ (теорема 3 ) и любом $n \geqslant 2$;

(5) не нарушается в $P_{2}$ (см. [5]).

Таким образом, гипотеза 2 полностью доказана в части необходимости $k$ быть простым для выполнения условия Голича в $P_{k}$. Кроме того, доказан ряд утверждений, косвенно подтверждающих справедливость гипотезы 2 в части достаточности.

\section{Список литературы}

1. Dichtl M., On nonlinear filter generators. Lecture Notes Computer Sci. (1997) 1267, 103-106.

2. Golić J., On the security of nonlinear filter generators. Lecture Notes Computer Sci. (1996) 1039, $173-188$.

3. Hedlund G. A., Endomorphisms and automorphisms of the shift dynamical system. Math. Syst. Theory (1969) 3, 320-375.

4. Logachev O. A., Salnikov A. A., Smyshlyaev S. V., Yashchenko V. V., Perfectly balanced functions in symbolic dynamics. In: Proc. NATO Advanced Research Workshop on Enhancing Cryptographic Primitives with Techniques from Error Correcting Codes. IOS Press, Amsterdam, 2009, pp. 222-233.

5. Smyshlyaev S. V., Perfectly balanced Boolean functions and Golić conjecture. J. Cryptology (2012) 25, 464-483.

6. Логачев О. А., Смышляев С. В., Ященко В. В., Новые методы изучения совершенно уравновешенных булевых функций. Дискретная математика (2009) 21, №2, 51-74.

7. Смышляев С. В., Булевы функции без предсказывания. Дискретная математика (2011) 23, №1, $102-118$.

8. Сумароков С. Н., Запреты двоичных функций и обратимость для одного класса кодирующих устройств. Обозрение прикладной и промышленной математики (1994) 1, №1, 33-55. 